\section{Altura de los tallados coronales en la retención de prótesis parcial fija}

Nogueira JL, Salazar Marocho SM, Pavanelli AC, Zamboni Costa S, Vasconcellos LG. Altura de los tallados coronales en la retención de prótesis parcial fija. Rev Estomatol Herediana. 2008; 18(2):114-117.

\section{RESUMEN}

La altura y convergencia de las paredes axiales, el diámetro de los tallados, la presencia de surcos para mejorar la retención y el tipo de cemento son algunas de las condiciones que influencian el ajuste y la retención de las restauraciones fundidas. El objetivo de este trabajo fue verificar la relación de la cantidad de retención con el aumento gradual de la altura de los tallados coronales. Usando 11 troqueles y 11 cápsulas metálicas, fresados y perfectamente adaptados, con alturas desde $3 \mathrm{~mm}$ hasta $8 \mathrm{~mm}$, con el aumento gradual de $0,5 \mathrm{~mm}$. Todas las capsuladas fueron cementadas de forma estandarizada y luego de 24 horas fueron sometidas a la prueba de tracción (EMIC 1000). Los resultados mostraron que incrementos de $0,5 \mathrm{~mm}$ en la altura del tallado aumentaron en promedio de $7 \mathrm{~N}$ la resistencia a tracción de los cuerpos de prueba, por tal motivo se puede concluir que existe una influencia de la altura del tallado en la capacidad retentiva de los troqueles.

Palabras clave: DENTADURA PARCIAL FIJA / CORONAS / RETENCIÓN DE PRÓTESIS DENTAL.

\section{Coronal preparation height on the retention of fixed partial dentures}

\section{ABSTRACT}

The height and convergence of axial walls, the diameter of the coronal preparation, the presence of grooves to improve retention and the type of cement are some of the conditions that influence the adjustment and retention of cast restorations. The aim of this study was to verify the relationship between the amount of retention with the gradual increase in the height of the coronal preparation. Using 11 die and 11 metal capsules milled and perfectly adapted, with heights from $3 \mathrm{~mm}$ to $8 \mathrm{~mm}$, with the gradual increase of $0.5 \mathrm{~mm}$. All capsules were cements in a standardized way, and after 24 hours were tested for tensile strength (EMIC-1000). The results showed increases of $0.5 \mathrm{~mm}$ in height of coronal preparations, rose $7 \mathrm{~N}$ on average the traction resistance of all tested bodies, therefore we can conclude that there is an influence of the height of the coronal preparation on the retention capacity of dies.

Key words: FIXED PARTIAL DENTURE / CROWNS / DENTAL PROSTHESIS RETENTION.

\section{Junior Lafayette Nogueira' Susana María Salazar Marocho² Augusto Carlos Pavanelli ${ }^{1}$ Sandra Zamboni Costa ${ }^{2}$ Luiz Gustavo Vasconcellos ${ }^{2}$}

'Docente del Departamento de Materiales Odontológicos y Prótesis.

${ }^{2}$ Magister en Odontología Restauradora - Área de Concentración: Prótesis Dental.

Facultad de Odontología de San José de los Campos - UNESP, Brasil.

\section{Correspondencia}

Junior Lafayette Nogueira

Francisco José Longo, 777, São Dimas

122 45000, São José dos Campos, SP. Brasil.

Teléfono: +55 12-39479061

e-mail: lafayette@ fosjc.unesp.br

Recibido : 13 de octubre del 2008

Aceptado : 15 de diciembre del 2008

\section{Introducción}

Varios son los factores que determinan el éxito o fracaso de una prótesis fija (PPF). La retención es uno de los factores más importantes. Cuando es insuficiente, es común en volverse la primera señal detectada por el paciente, lo que sugiere que el tratamiento fracasó. En este caso, fuerzas mínimas oclusales hacen que el agente de cementación se rompa y la prótesis se disloque del diente tallado.

Varios factores afectan la retención de una pieza protética y han sido agrupados en tres categorías por Kaufman et al. (1961) (1): tallado del diente, tipo de metal y material de cementación. La geometría de los tallados, en este caso, puede ser considerada como un factor que limita e influencia la cantidad de retención.

La forma, conicidad del tallado y el área de dentina envuelta (altura y ancho del remanente dental), pueden hasta cierto punto ser manipulados por el criterio clínico conforme la necesidad de cada caso.

La flexibilidad de la decisión del profesional es importante y deseable, porque permite muchas veces, la ejecución de procedimientos más conservadores y la preservación de las estructuras. Así, pueden ser citados los casos de prótesis unitarias, donde hay proporcionalmente menor necesidad de retención, si es comparado con las PPF extensas con varios dientes pilares (Shillingburg et al. 1998) (2). Seria temeraria una estandarización didáctica de los valores de retención necesaria, solamente considerando el tipo de tallado coronal.

Parece ser más coherente que la altura final posible del tallado, sea condicionada a la altura natural del diente de soporte y que el grosor requerido para la obtención de una forma de resistencia sea adecuada al material restaurador.

La literatura constata una estrecha relación entre la altura de los tallados, área de superficie de contacto diente-restauración y retención (2-6). Pero, también es común observar situaciones en la que los autores estudian una gran cantidad de variables al mismo tiempo, generando muchas veces resultados confusos.

Sin embargo, parece haber concordancia que aumentar el área de contacto entre la superficie interna de la corona y la superficie dentinaria del tallado, aumenta la retención de la prótesis. Como forma de aumentarse el área de contacto, Kaufman et al. (1), en 1961, relacionaron el aumento de la altura, ancho, confección de surcos, postes y el tipo de acabado de las superficies.

La altura del tallado es probable- 
mente el factor que más puede estar sujeto a variaciones, causando sensibles variaciones en la cantidad de retención de la prótesis. Siendo así, sería deseable que pudiésemos establecer una relación de proporcionalidad entre altura y retención.

A partir de pruebas controladas en laboratorio, se realizó la tracción para el desprendimiento de cápsulas metálicas cementadas sobre troqueles también metálicos, teniendo como única variable la altura del tallado.

El objetivo de este trabajo fue verificar la relación de la cantidad de retención generada, con el aumento gradual en la altura de los tallados coronales.

\section{Material y métodos}

Se obtuvieron once troqueles y once cápsulas en acero inoxidable, que eran representativos de los tallados para coronas totales y sus mismas coronas, a través del fresado de cilindros de acero inoxidable, en un torno mecánico industrial de precisión. El mayor diámetro (cervical) de cada troquel fue de $8 \mathrm{~mm}$, con expulsividad de $10^{\circ}$ para oclusal (7) y línea de terminación cervical en forma de filo de cuchillo.

Todos los troqueles fueron fresados con medidas iguales, excepto a la altura, en la cual el troquel 1 fue de $3 \mathrm{~mm}$, incrementado $0,5 \mathrm{~mm}$ sucesivamente, hasta el troquel 11 , donde la altura fue de $8 \mathrm{~mm}$. Cada troquel recibió un alivio de $0,025 \mathrm{~mm}$ en su superficie externa (8-12), también por fresado, a partir de la línea de terminación, excepto en el primer milímetro cervical, próximo a la línea de terminación.

Las cápsulas metálicas fueron fresadas, de forma individual, perfectamente adaptadas a sus respectivos troqueles (preservando el alivio predeterminado) e identificadas por numeración de 1 a 11. Se utilizó cemento de fosfato de zinc Lee Smith (Teledyne, Brasil), para todas las cementaciones, siguiendo las instrucciones del fabricante con respecto a proporción polvo-líquido (orientación de la ADA, especificación $\mathrm{N}^{\circ} 8$ para cementos de fosfato de zinc) (13). Para la manipulación del cemento se utilizaron placas de vidrio enfriadas $(11,14)$.

Para disminuir la espesura de la película de cemento y mejorar la adaptación marginal, el cemento fue aplicado al interior de las cápsulas con un pincel $(10,15)$, seguida de la aplicación de presión de cementación estática de $5 \mathrm{Kgf}$ sobre cada uno de los once conjuntos cápsula-troquel $(15,16)$ (denominado cuerpos de prueba). Esa presión fue dada por un aparato apropiado (17), calibrado para esa finalidad, durante un minuto.

Después del período mínimo de 24 horas $(13,14,18)$, los cuerpos de prueba fueron sometidos a la prueba de resistencia a tracción con auxilio de un equipo para prueba de tracción EMIC - 1000 y una máquina universal de ensayos - Itaquá SP - Brasil del LAP (Laboratorio de apoyo a la Investigación de la Facultad de Odontología de São José dos Campos - UNESP). Los valores obtenidos fueron anotados y transferidos a una tabla representativa de la primera serie de cementaciones. Estos procedimientos, hasta las pruebas de tracción, fueron repetidos por cuatro veces más de forma idéntica, para que se obtenga el valor promedio de los cinco resultados.

Entre una y otra serie de pruebas, la remoción del cemento y la limpieza de los troqueles y capsulas fue hecha por baño en ultrasonido con una solución de detergente, hasta la total desintegración del cemento (12).

\section{Resultados}

La figura 1 muestra los valores en Newtons, de la fuerza necesaria para la separación de los cuerpos de prueba en los cincos tests hechos con cada conjunto. Se calculó enseguida el promedio aritmético de los valores obtenidos, estableciendo el valor promedio de la fuerza necesaria de retención entre otros tipos de talla para dicha separación. Podemos

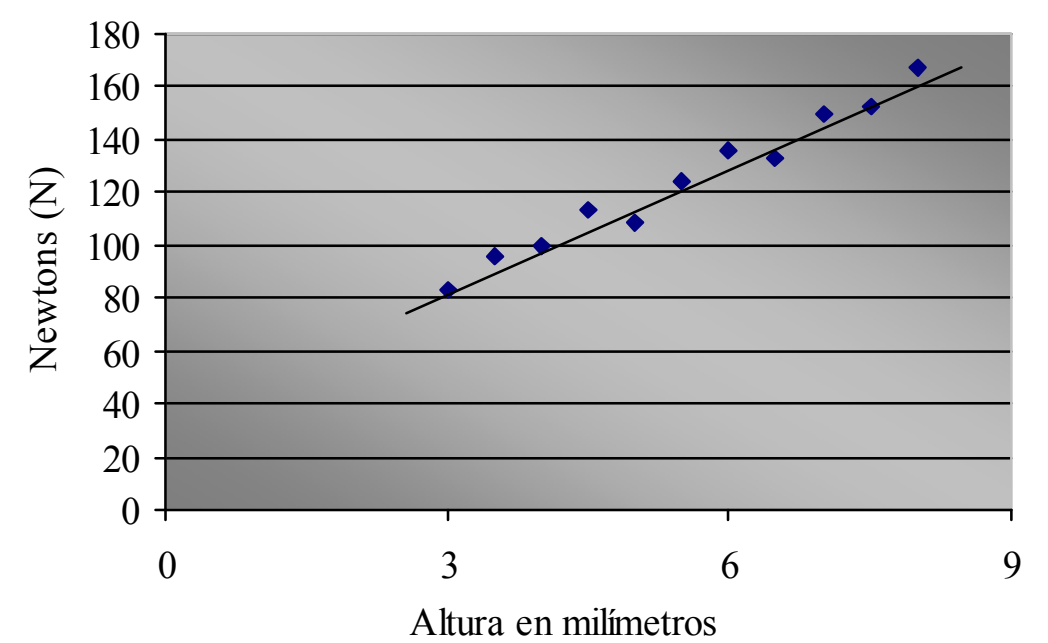

Fig. 1. Mostrando la linealidad de crecimiento de la fuerza necesaria al arrancamiento de la cápsula del cuerpo de prueba (en Newtons) en función del aumento de la altura del tallado (en milímetros). 
notar una influencia linear de la altura del tallado en la capacidad retentiva de los troqueles. Trazando una línea entre el valor menor y mayor, se puede observar una cierta linea de crecimiento de los valores.

En la figura 2 se muestra la variación de la capacidad de retención de cada cuerpo de prueba mientras la altura del troquel aumenta y el ángulo de convergencia permanece constante.

\section{Discusión}

El uso de coronas totales o PPF para restaurar o reponer dientes ha sido mostrado a lo largo del tiempo, como un procedimiento duradero, dentro de condiciones próximas de la ideal. Con el refinamiento de técnicas y la actualización de los métodos de laboratorio para la confección de restauraciones metálicas fundidas, es importante entender los fundamentos que implican la retención de las restauraciones metálicas cementadas.

Los factores o propiedades que parecen afectar la capacidad de retención de una pieza protésica, hace mucho han sido reportados. Sin embargo, pocos trabajos determinan cualitativamente la capacidad retentiva de un tallado coronario y su restauración. Muchos dientes han sido cuestionados en relación a su posibilidad de uso como retenedores de PPF, solamente con base a la altura coronaria mínima o área del tallado.

Observamos en la literatura, concordancia, en que muchos factores influencian la capacidad de retención de un tallado coronario, entre ellos: área total de superficie, altura de la superficie preparada, grado de convergencia de las paredes opuestas del tallado, textura de la superficie preparada y artificios de retención intracoronarios (19).

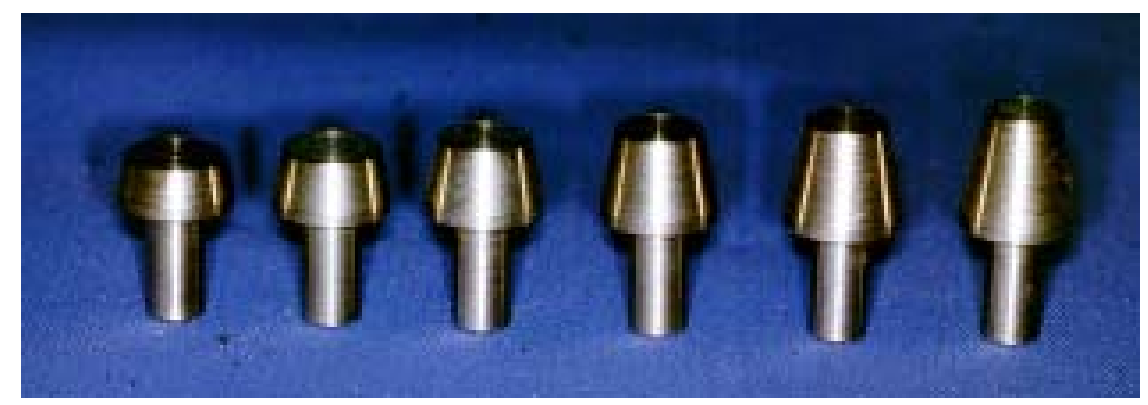

Fig. 2. Muestra de los tallados coronales y coronas metálicas.

Relacionados a los factores que pueden afectar la retención, influenciados por la fundición encontramos: adaptación de la fundición al diente preparado, textura de la superficie interna de la fundición y las propiedades físicas del metal necesarias para el mantenimiento del sellado marginal.

Existen también los factores relacionados al cemento que podrían ser agrupados en: tipo de cemento, el efecto de una abertura planeada en la restauración, su viscosidad, fuerza ejercida durante la cementación tiempo entre la cementación y procedimientos de remoción de la restauración, ángulo de la fuerza de remoción y los valores de la resistencia a la compresión y cizallamiento de los diversos tipos de cementos.

En este trabajo se analizó la influencia de la altura de superficie preparada en la capacidad de retención de las coronas totales metálicas. Los tests realizados fueron conducidos bajo control rígido tanto de las partes coronarias como de las cápsulas metálicas representativas de las coronas totales. Los resultados obtenidos con la metodología usada es similar con la opinión de diversos autores, esto es: la capacidad retentiva de tallados coronarios está directamente relacionada con la altura de este tallado.

Para Rosenstiel et al.(20), 1988, la geometría del tallado dental tiene una relación íntima con la capaci- dad de retención y la forma de resistencia de la pieza protésica. Así, indicaron que el tallado coronario debería ser realizado de tal forma que incorpore otros recursos de retención en sus paredes axiales para dificultar la rotación de la restauración. Según esos autores, un tallado coronario corto con diámetro ancho ofrece pequeña capacidad de retención, con lo que concordamos.

Fusayama et al. (21), 1964, afirmaron que la presión de asentamiento en la cementación, la convergencia de las paredes axiales, la altura de los tallados y la cantidad de alivio de las coronas, influencian la adaptación de las coronas totales durante la cementación. Esto debe ser considerado, pues la retención sin adaptación fatalmente significaría fracaso del trabajo protésico.

Lorey y Myers (22), 1968, encontraron que el tallado de corona total proporcionó un valor máximo dos (coronas tres cuartos, coronas tres cuartos con postes y dos tipos de tallados tipo pin ledge), comprobando que la retención está directamente relacionada al área de superficie dentaria utilizada en el tallado.

Los resultados obtenidos por Maxwell et al. (23) en 1990, indicando tallados con un mínimo de tres milimetros de altura, nos orientaron a la confección del troquel 1 y nos estimularon a verificar la influencia de alturas mayores en la retención, hasta un máximo de $8 \mathrm{~mm}$, como fue citado por la mayoría de los demás 
autores consultados.

Shillingburg et al. (2) 1998, afirman que la altura del tallado puede influenciar la resistencia al dislocamiento de las piezas protéticas. Para esos autores, el tallado coronario bajo presentará, proporcionalmente, disminución en la resistencia a la tracción. Pero, la capacidad de la restauración de resistir al dislocamiento después de la cementación, no depende solamente del tallado, sino también de la magnitud y dirección del torque. Tenemos que estar de acuerdo con esa afirmación, principalmente porque a pesar de que los tallados muchas veces poseen la misma altura, generalmente sus coronas, para cumplir los requisitos de oclusión, pueden presentar alturas diferentes y con eso estarían sometidas a formas diferentes de carga.

\section{Conclusiones}

- La altura del tallado coronal influenció en la capacidad de retención de las coronas totales.

- La influencia del aumento de la altura del tallado ocurrió de forma aproximadamente lineal y creciente con la altura.

- Aumentos de $0,5 \mathrm{~mm}$ en la altura, aumentaron en promedio de $7 \mathrm{~N}$ la resistencia a tracción de los cuerpos de prueba.

\section{Referencias bibliográficas}

1. Kaufman EG, Colin L, Schlagel E, Coelho DH. Factors influencing the retention of cemented gold castings: The cementing medium. J Prosthet Dent. 1966; 16(4):731-9.

2. Shillingburg HT, Hobo S, Whitsett LD. Fundamentos de prostodoncia fija. 2da Ed. Chicago: Quintessence Books; 1978.

3. Douglass GD. Principles of preparation design in fixed prosthodontics. J Acad Gen Dent. 1973;21(2):25-9.

4. Rosenstiel SF. Contemporary fixed prosthodontics. St. Louis: Mosby-Year Book; 1988.

5. Saito T. Preparos dentais funcionais em prótese fixa. São Paulo:Quintessence Books; 1994.

6. Maxwell AW, Blank LW, Pelleu GB Jr. Effect of crown preparation height on the retention and resistance of gold castings. Gen Dent. 1990; 38(3):200-2.

7. Jørgensen KD. The relationship between retention and convergence angle in cemented veneer crowns. Acta Odontol Scand. 1955; 13(1):35-40.

8. Bassett RW. Solving the problems of cementing the full veneer cast gold crown. J Prosthet Dent. 1966; 16(4):740-7.

9. Christensen GJ. Cimentos, agentes de forramento e bases em prótese fixa. En: Malone WFP, Koth DL, Teoria e prática de prótese fixa de Tylman. 8a ed. São Paulo: Ed. Artes Médicas; 1991.

10. Ishikiriama A, Oliveira Jde F, Vieira DF, Mondelli J. Influence of some factors on the fit of cemented crowns. J Prosthet Dent. 1981; 45(4):400-4.

11. Jørgensen KD. Factors affecting the film thickness of zinc phosphate cements. Acta Odontol Scand. 1960; 18(4):479-90.

12.Vieira DF. Cimentação, incrustações, coroas e próteses fixas. São Paulo: Savier; 1976.

13.Phillips RW. Cimentos para cimentação. En: Phillips RW. Skinner materiais dentários. 9 ed. Rio de Janeiro: Guanabara Koogan; 1993.
14. Cimentos dentários para fixação. In:_ . Materiais dentários de Skinner. 8ed. Rio de Janeiro: Guanabara Koogan, 1986. Cap.29, p.330-50.

15. Eames WB, O'Neal SJ, Monteiro J, Miller C, Roan JD Jr, Cohen KS. Techniques to improve the seating of castings. J Am Dent Assoc. 1978; 96(3):432-7.

16. Hoard RJ, Caputo AA, Contino RM, Koenig ME. Intracoronal pressure during crown cementation. J Prosthet Dent. 1978; 40(5):520-5.

17.Paffenbarger GC, Sweeney WT, Isaacs A. Zinc phosphate cements: Physical properties and a specification. J Am Dental Assoc. 1934; 21:1907-24.

18.Gegauff AG, Rosenstiel SF. Reassessment of die-spacer with dynamic loading during cementation. J Prosthet Dent. 1989; 61(6):655-8.

19 Vieria DF. Cimentação: incrustações, coroas e próteses fixas. São Paulo: Sarvier; 1976.

20 Rosenstiel SF, Gegauff AG. Improving the cementation of complete cast crowns: a comparison of static and dynamic seating methods. J Am Dent Assoc. 1988; 117(7):845-8.

21 Fusayama T, Ide K, Hosada H. Relief of resistance of cement of full cast crowns. J Prosthet Dent. 1964; 14:95-106.

22 Lorey RE, Myers GE. The retentive qualities of bridge retainers. J Am Dent Assoc. 1968; 76(3):568-72.

23 Maxwell AW, Blank LW, Pelleu GB Jr. Effect of crown preparation height on the retention and resistance of gold castings. Gen Dent. 1990; 38(3):200-2. 